\title{
Propagação de incertezas usando planilhas eletrônicas
}

\section{Propagation of uncertainties using spreadsheets}

\author{
Rodrigo P. R. Figueiredo $\stackrel{10}{ }$, George C. Cardoso*1] \\ ${ }^{1}$ Universidade de São Paulo, Departamento de Física, FFCLRP, Ribeirão Preto, SP, Brasil.
}

Recebido em 18 de fevereiro de 2021. Revisado em 04 de julho de 2021. Aceito em 15 de julho de 2021.

\begin{abstract}
Propomos uma metodologia de propagação de incertezas por diferenças finitas para atividades de laboratório de ensino. O público-alvo da abordagem proposta são alunos que já adquiriram alguma maturidade na teoria de medidas e propagação de incertezas experimentais. O próprio aluno poderá implementar a metodologia em planilhas eletrônicas para análise de dados e propagação de incertezas. Visamos assim aumentar o controle e sofisticação do processamento dos dados pelo aluno, diminuir sua carga cognitiva e o tempo gasto na tarefa. Dessa forma, outras etapas também importantes da atividade de laboratório, incluindo planejamento, análise, e entendimento dos resultados e limitações do experimento, poderão ser desenvolvidas com mais cuidado.
\end{abstract}

Palavras-chave: Incertezas experimentais, propagação de incertezas, laboratório de ensino.

We propose a methodology for uncertainty propagation by finite differences for teaching laboratory activities. The public for the proposed approach is students who already have some maturity in measurement theory and experimental uncertainty propagation. The students themselves may implement the methodology in electronic spreadsheets for data analysis and uncertainty propagation. This way, we hope to increase student control and sophistication in data processing, and to decrease their cognitive load and time spent in the task. Thus, other also important parts of the laboratory assignments, such as planning, analysis, and understanding the results and limitations of the experiment, may be more carefully developed.

Keywords: Experimental uncertainties, Propagation of uncertainties, Teaching laboratory.

\section{Introdução}

Medidas, incertezas, e propagação de incertezas experimentais através de funções matemáticas são conteúdos fundamentais nas disciplinas básicas de laboratório de ciências naturais e exatas [1, 2]. Entretanto, o ensino convencional de propagação de incertezas experimentais exige conhecimento básico de derivadas parciais 3,4 . Frequentemente, o aluno de primeiro ano não está matematicamente preparado e fica refratário em relação à aplicação da teoria de medidas [5] 7]. Almejamos diminuir a aversão contra as disciplinas experimentais, em particular de alunos que não são de física, ao oferecer uma alternativa ao formalismo que exige derivar para propagar incertezas 8, 9 .

Neste artigo apresentamos um método para propagação de incertezas experimentais por diferenças finitas que evita o uso de derivadas e permite implementação em planilhas eletrônicas. Sugerimos que nas atividades onde o foco do ensino não sejam incertezas experimentais, automação do cálculo da propagação incertezas beneficia o aprendizado porque reduz a carga cognitiva do aluno. Isso o permite se concentrar na apresentação correta dos resultados tais como algarismos significativos, e no entendimento e discussão do experimento. A compatibilidade da metodologia proposta com

\footnotetext{
*Endereço de correspondência: gcc@usp.br
}

planilhas eletrônicas permite integrar todas as análises no mesmo software onde os dados são tipicamente registrados.

\section{Incertezas de medição}

Medir consiste em determinar características quantitativas de uma grandeza, o que normalmente corresponde a estimar a estatística de uma distribuição de valores de leituras de um mensurando com uma dada metodologia experimental. As propriedades mais simplificadoras de uma estatística são o valor médio aritmético, quando existe $\rrbracket^{1}$ e sua mediana. Assim, em primeira aproximação, medir é estimar o valor médio ou mediano verdadeiro da grandeza que seria estritamente obtido somente incluindo um número infinito de leituras na estatística. Neste artigo usaremos como valor medido a estimativa do valor médio das leituras, adequado para a maioria das medidas típicas, onde a média tende a seu valor verdadeiro à medida que o número de leituras aumenta. Porém, na prática, o número de leituras é sempre finito impossibilitando a obtenção do valor médio verdadeiro. Para quantificar a falta de conhecimento do valor médio

\footnotetext{
1 Para distribuições estatísticas tais como a de Cauchy ou de Breit-Wigner (curva Lorentizana) a média é indefinida, pois não converge. É preciso usar a mediana.
} 
verdadeiro da medida, utilizamos o conceito de incerteza de medição.

Incerteza de medição não se refere a erros operacionais ou problemas metodológicos. Flutuações aleatórias nas leituras são inevitáveis e intrínsecas de qualquer processo de medida. Para estimar incerteza, múltiplas leituras são necessárias em uma medição. A dispersão de valores observados pode ser visualizada em um histograma que, com o aumento do número de leituras, tende à distribuição estatística da variável medida com a metodologia adotada. A largura desse histograma se relaciona com a variabilidade no valor do mensurando e sua incerteza estatística.

Sempre que escrevemos uma incerteza, precisamos especificar sua definição sem ambiguidade. Porque os principais usos de incerteza de medição são determinar o número de algarismos significativos na apresentação do valor medido, e estimar concordância ou discrepância entre o valor medido e outro valor medido ou esperado. Convencionalmente, escreve-se um resultado de medida experimental como $\bar{x} \pm \alpha$, onde $\bar{x}$ representa o valor médio do mensurando $x$, e $\alpha$ é a incerteza correspondente, cuja definição precisa ser especificada. As incertezas de medição tipicamente usadas são desvios padrões e incertezas padrões (desvios padrões da média), ambas incertezas estatísticas.

O desvio padrão (DP) é a forma mais usada para medir incertezas. Como em uma medida o número $N$ de leituras é finito, o que se pode determinar é a estimativa do DP (DP amostral, s):

$$
s=\left(\frac{1}{N-1} \sum_{i=1}^{N}\left(x_{i}-\bar{x}\right)^{2}\right)^{1 / 2}
$$

onde $x_{i}$ é o valor lido na $i$-ésima observação, e $\bar{x}$ é a estimativa do valor médio do mensurando $x$ obtida com as $N$ leituras. DP é uma característica combinada do sistema de medida com o mensurando. No laboratório de ensino de física, o mensurando tipicamente se mantém constante durante as medições e o DP é uma característica apenas da metodologia de medida, um exemplo de mensurando constante é o comprimento de uma mesa. O DP, estimado por $s$, equação (1), é equivalente ao valor eficaz da flutuação das leituras em torno do valor médio (valor quadrático médio, RMS), e converge para uma constante $\sigma$ para $N$ grande. O valor $\sigma$ é uma característica combinada da metodologia de medida e do mensurando. $\mathrm{O}$ valor $s^{2}$, chamado variância da amostra, é proporcional à potência média do ruído da medida [10].

A interpretação do DP depende da distribuição estatística das leituras na medida. Por exemplo, na medida:

$$
L=(2,11 \pm 0,19) \mathrm{m}(1 \mathrm{DP}, \mathrm{N}=80 \text { medidas })
$$

temos a incerteza de 1 DP. Se a estatística da distribuição das leituras for gaussiana, $f=68 \%$ das leituras ficam no intervalo entre $(2,11-0,19) \mathrm{m} \mathrm{e}$
$(2,11+0,19) \mathrm{m}$. Para distribuições não gaussianas (uniforme, lognormal, Poisson, etc.), a fração $f$ muda para um dado intervalo de \pm 1 DP. A rigor a distribuição estatística das medidas precisa ser estimada. Porém, para simplificar, no laboratório de ensino assumimos distribuições gaussianas - mais encontradas experimentalmente. Para uma interpretação mais cuidadosa, a necessidade do conhecimento da distribuição estatística das variáveis ou da distribuição de uma função após propagação das variáveis, é uma desvantagem do uso do DP para descrever incertezas. Em particular, mesmo quando variáveis têm distribuições estatísticas gaussianas, mas a função é não-linear nessas variáveis, a distribuição dos valores da função será não-gaussiana quando incertezas grandes são propagadas. Contudo, o DP é a incerteza mais comumente utilizada, e é especialmente adequado para descrever a incerteza da metodologia de medida, ou das flutuações médias do próprio mensurando, ou ambos.

O desvio padrão da média (DPM) é outra descrição para incertezas. O DPM é também conhecido como incerteza padrão, antigamente chamado erro padrão. Descreve a flutuação no valor médio entre repetições do experimento com mesma metodologia e número $\mathrm{N}$ de leituras ou observações. O DPM é calculado por:

$$
\sigma_{\bar{x}}=\frac{s}{\sqrt{N}}
$$

onde $\sigma_{\bar{x}}$ é o DPM, $s$ é o DP estimado (equação 1). Teoricamente, usando uma mesma metodologia, duas medidas independentes do mesmo observável obterão o mesmo valor médio - o valor médio verdadeiro - quando em ambas medidas o número $N$ de leituras tender a infinito. Portanto, o DPM tende a zero quando $N$ tende a infinito, em contraste com o DP que é uma constante e depende do sistema de medida e do mensurando.

O DPM tem a vantagem de sempre apresentar uma distribuição gaussiana (para $N$ grande), mesmo que a distribuição estatística das medidas seja nãogaussiana [10]. Vejamos como o valor de $L$, mostrado no exemplo acima com DP, fica quando descrito por DPM:

$$
L=(2,11 \pm 0,02) \mathrm{m}, \mathrm{IC} 68 \%,
$$

onde o IC indica intervalo de confiança e em repetições do experimento de medida de $L, 68 \%$ dos valores médios encontrados estarão entre $(2,11-0,02) \mathrm{m}$, e $(2,11+0,02) \mathrm{m}$. Para melhorar a estimativa, usamos tipicamente IC 95\%. Para isso, para $N \geq 15$, uma boa aproximação é usar $2 \mathrm{DPM}^{2} L=(2,11 \pm 0,04) \mathrm{m}$, IC $95 \%$. Significa que, por exemplo, em uma turma com 20 grupos experimentais, em média $5 \%$ dos grupos (1 grupo) obterá um resultado fora do IC de $95 \%$ caso

\footnotetext{
2 Para $N$ menor, o fator multiplicador aumenta. No caso geral, para $N \geq 2$, o IC de $95 \%$ é obtido multiplicando-se o DPM pelo inverso bicaudal da distribuição t de Student (TINV( ), em planilhas MS Excel e OpenOffice), com $\mathrm{p}=5 \%$, e $(N-1)$ graus de liberdade.
} 
o mesmo equipamento, o mesmo objeto medido, e a mesma metodologia sejam utilizados. O DPM descreve a incerteza do valor médio, e não o valor eficaz das flutuações como o DP. Assim, em física e engenharia o DPM é mais útil. Além do DPM sempre apresentar distribuição gaussiana, o que simplifica na prática.

O DP e o DPM são incertezas estatísticas, chamadas incertezas do tipo $A$. Existem ainda incertezas instrumentais, chamadas do tipo $B$, devidas a limitações de resolução ou de calibração da instrumentação de medida. A incerteza total de uma medida é a soma em quadratura das incertezas do tipo $\mathrm{A}$ e do tipo $\mathrm{B}$, ou seja: $\sigma_{T}^{2}=$ $\sigma_{A}^{2}+\sigma_{B}^{2}$. Neste artigo, nos concentramos em incertezas do tipo A.

\section{Propagação de incertezas}

Nesta seção derivamos uma expressão para propagação de incertezas por diferenças finitas. Todas as fórmulas usadas aqui são válidas tanto para desvios padrões (DP) quanto para incertezas padrões (DPM), desde que a mesma fórmula contenha somente incertezas de mesma definição e intervalos de confiança. Assim, no que se segue omitiremos as especificações do que chamaremos simplesmente de incertezas. Lembremos que para valores numéricos precisamos sempre especificar o significado das incertezas, como nos exemplos da seção anterior.

Ilustramos o problema de propagação de incertezas com um exemplo de pêndulo simples. Ao final desta seção, generalizamos a discussão para uma função qualquer. No pêndulo simples, para pequenas oscilações, determinamos a aceleração $g$ da gravidade através de [11]

$$
g(L, T)=4 \pi^{2} \frac{L}{T^{2}}
$$

onde $L$ é a medida do comprimento do fio do pêndulo e $T$ é a medida do seu período de oscilação. Experimentalmente, fazem-se leituras repetidas das variáveis $L$ e $T$ para determinar $\bar{L} \pm \sigma_{L}$ e $\bar{T} \pm \sigma_{T}$, onde a barra sobre as variáveis indica valores médios, e $\sigma_{L}, \sigma_{T}$ representam as incertezas [10. Um dos objetivos acadêmicos do experimento do pêndulo simples é determinar o valor médio esperado para $g(L, T)$ e sua respectiva incerteza $\sigma_{g}$, consequência das incertezas nos parâmetros $L$ e $T$ [12, 13.

No laboratório de ensino, $L$ e $T$ são mensurados de forma independente para ficarem descorrelacionados, e não apresentarem covariância [10]. Nessa condição, a variância $\sigma_{g}^{2}$ é a soma das variâncias de $g$ devidas às flutuações estatísticas nas leituras das variáveis $L$ e $T$ :

$$
\sigma_{g}^{2}=\sigma_{g(L, \bar{T})}^{2}+\sigma_{g(\bar{L}, T)}^{2}
$$

que é uma soma em quadratura das incertezas parciais devidas a cada uma das variáveis. Para $\sigma_{L} / \bar{L}$, e $\sigma_{T} / \bar{T}$ muito pequenos, $g(L, T)$ em torno do ponto $(\bar{L}, \bar{T})$ fica aproximadamente linear nas variáveis $L$ e $T$ (Exemplo na seção 4). Podemos então usar derivadas para determinar o efeito das pequenas variações $\sigma_{L}$, e $\sigma_{T}$ nos termos do lado direito da equação (4) [3]:

$$
\sigma_{g}^{2} \approx\left(\frac{\partial g}{\partial L} \sigma_{L}\right)^{2}+\left(\frac{\partial g}{\partial T} \sigma_{T}\right)^{2}
$$

onde $\partial g / \partial L=d g(L, \bar{T}) / d L$ é a derivada parcial de $g(L, T)$ em relação a $L$, e $\partial g / \partial T$ é análogo. Na prática do ensino, calculam-se as derivadas de forma tradicional, substituem-se as expressões das derivadas na equação (5), e finaliza-se o cálculo usando calculadora ou planilha eletrônica para determinar $\sigma_{g}$.

Podemos citar imediatamente duas desvantagens no uso rotineiro da equação (5) no âmbito das atividades de laboratório de ensino. A primeira, são as derivadas parciais. Porque alguns alunos ainda estão pouco familiarizados com o cálculo diferencial. A segunda, são os possíveis erros operacionais na transcrição das derivadas obtidas para a equação (5), mesmo que as derivadas parciais tenham sido calculadas corretamente. A relativa complexidade operacional da equação (5) depende da descrição matemática do fenômeno físico em estudo. Erros de derivação e de digitação das expressões encontradas são de difícil identificação por inspeção do valor obtido para $\sigma_{g}$.

Vamos agora explicar a metodologia para propagação de incertezas por diferenças finitas. Por definição de derivadas, $\partial g / \partial L=\Delta g / \Delta L$, para $\Delta L$ muito pequeno. Usando $\sigma_{L}$, que assumimos ser muito pequeno e que tem dimensão de $L$, no lugar de $\Delta L$ no denominador de $\Delta g / \Delta L$, a derivada parcial fica $\Delta g / \sigma_{L}$. Usando esse artifício no primeiro termo do lado direito da equação (5), ficamos apenas com a variação $\Delta g$ causada por uma variação $\sigma_{L}$. Usando raciocínio análogo para o segundo termo da equação (5), obtemos uma equação de diferenças finitas:

$$
\begin{aligned}
\sigma_{g}^{2} \approx & \left|g(\bar{L}, \bar{T})-g\left(\bar{L} \pm \sigma_{L}, \bar{T}\right)\right|^{2} \\
& +\left|g(\bar{L}, \bar{T})-g\left(\bar{L}, \bar{T} \pm \sigma_{T}\right)\right|^{2}
\end{aligned}
$$

No limite em que $\sigma_{L}$ e $\sigma_{T}$ são pequenos o resultado da (5) é idêntico ao da equação (6). Porém, enquanto a (5) é uma aproximação linear rigorosamente correta apenas para incertezas infinitesimais, a equação (6) é mais robusta e permite incertezas finitas.

Análogo à equação (6), para uma função $f(x, y)$ qualquer temos:

$$
\begin{aligned}
\sigma_{f}^{2} \approx & \left|f(\bar{x}, \bar{y})-f\left(\bar{x} \pm \sigma_{x}, \bar{y}\right)\right|^{2} \\
& +\left|f(\bar{x}, \bar{y})-f\left(\bar{x}, \bar{y} \pm \sigma_{y}\right)\right|^{2}
\end{aligned}
$$

onde $\bar{x}, \bar{y}$ são os valores médios dos mensurandos, e $\sigma_{x}, \sigma_{y}$, suas respectivas incertezas. Para valores não desprezíveis de $\sigma_{x}, \sigma_{y}$, o valor de $\sigma_{f}$ pode depender do sinal $( \pm)$ utilizado. Essa assimetria na incerteza [14] desaparece para $\sigma_{x}, \sigma_{y}$ pequenos, ou se a incerteza 
Tabela 1: Esquema para implementação da propagação de incertezas por diferenças finitas em planilhas. As quatro primeiras colunas são auxiliares. A coluna $\Delta f^{S}$ contém os dois termos para uso no lado direito da equação 9

\begin{tabular}{|c|c|c|c|c|}
\hline$x$ & $y$ & $f(x, y)$ & $\Delta f$ & $\Delta f^{S}$ \\
\hline $\bar{x}$ & $\bar{y}$ & $f(\bar{x}, \bar{y})$ & 0 & \\
\hline $\bar{x}+\sigma_{x}$ & $\bar{y}$ & $f\left(\bar{x}+\sigma_{x}, \bar{y}\right)$ & $\Delta f_{x}^{+}=\left|f\left(\bar{x}+\sigma_{x}, \bar{y}\right)-f(\bar{x}, \bar{y})\right|$ & $\Delta f_{x}^{+}+\Delta f_{x}^{-}$ \\
\hline $\bar{x}-\sigma_{x}$ & $\bar{y}$ & $f\left(\bar{x}-\sigma_{x}, \bar{y}\right)$ & $\Delta f_{x}^{-}=\left|f\left(\bar{x}-\sigma_{x}, \bar{y}\right)-f(\bar{x}, \bar{y})\right|$ & 2 \\
\hline $\bar{x}$ & $\bar{y}+\sigma_{y}$ & $f\left(\bar{x}, \bar{y}+\sigma_{y}\right)$ & $\Delta f_{y}^{+}=\left|f\left(\bar{x}, \bar{y}+\sigma_{y}\right)-f(\bar{x}, \bar{y})\right|$ & $\Delta f_{y}^{+}+\Delta f_{y}^{-}$ \\
\hline $\bar{x}$ & $\bar{y}-\sigma_{y}$ & $f\left(\bar{x}, \bar{y}-\sigma_{y}\right)$ & $\Delta f_{y}^{-}=\left|f\left(\bar{x}, \bar{y}-\sigma_{y}\right)-f(\bar{x}, \bar{y})\right|$ & 2 \\
\hline
\end{tabular}

precisa ser escrita com poucos algarismos significativos ou para uma combinação dessas condições.

Para incertezas pequenas e funções monotônicas em torno de $(\bar{x}, \bar{y})$, podemos evitar escrever incertezas assimétricas calculando, para cada termo da equação (7), a média das incertezas obtidas com sinais positivos e negativos. Tal simetrização é prática comum na literatura [15, 16. Assim o primeiro termo do lado direito da equação (7) fica:

$\Delta f_{x}^{S} \approx \frac{\left|f\left(\bar{x}+\sigma_{x}, \bar{y}\right)-f(\bar{x}, \bar{y})\right|+\left|f\left(\bar{x}-\sigma_{x}, \bar{y}\right)-f(\bar{x}, \bar{y})\right|}{2}$,

onde o sobrescrito $S$ em $\Delta f_{x}^{S}$ indica incertezas simetrizadas, e o subscrito $x$ em $\Delta f_{x}^{S}$ indica o primeiro termo do lado direito da equação (7), onde a diferença finita é na variável $x$. Construindo uma expressão análoga $\Delta f_{y}^{S}$, a incerteza em $f(\bar{x}, \bar{y})$ dada pela equação (7) fica:

$$
\sigma_{f}^{2} \approx\left[\Delta f_{x}^{S}\right]^{2}+\left[\Delta f_{y}^{S}\right]^{2} .
$$

A Tabela 1 mostra um passo-a-passo para implementação da equação $(9)$ em planilha eletrônica. Generalizando a equação 91 para $N$ variáveis, um análogo à Tabela 1 terá $2^{N}$ combinações de incertezas.

\section{Exemplo em planilha eletrônica}

Implementamos a Tabela 1 e a equação $(9)$ em planilha eletrônica (Material suplementar). Na implementação em planilha, basta escrever $f(x, y)$ uma única vez e copiá-la para as células inferiores. Isso permite encontrar rapidamente possíveis erros, evitando o cálculo incorreto das incertezas. Por exemplo, no pêndulo simples sabemos que o valor da aceleração da gravidade é cerca de $g=$ $10 \mathrm{~m} / \mathrm{s}^{2}$. Se $f(x, y)$ for digitada errada, por exemplo, é possível que o valor de $f(\bar{x}, \bar{y})$ fique discrepante, revelando um erro grosseiro.

Comparamos agora a metodologia de diferenças finitas com a tradicional. Usaremos valores de um experimento simulado de pêndulo simples onde $L=(2,1 \pm 0,2) \mathrm{m}$ e $T=(3,00 \pm 0,10) \mathrm{s}$, com médias calculadas de 80 leituras simuladas, e incertezas representando DPs. Esses DPs são bem maiores que os tipicamente encontrados em laboratórios de ensino. Mesmo assim, observamos que a incerteza na função $g$ é idêntica para ambas metodologias, para um ou dois algarismos significativos na incerteza de $g$ (Figura 1). Lembremos que devido à incerteza da própria incerteza ${ }^{3}$ para experimentos com menos do que milhares de leituras, a incerteza no resultado deve ser arredondada para apenas um ou dois algarismos significativos [10].

Para esclarecer equivalência entre as duas técnicas, mostramos na Figura 2 como uma pequena incerteza em $\bar{T}$ projeta uma incerteza em $g(T)$. Indicamos as incertezas em torno de $\bar{T}=3,00$ s por linhas pontilhadas verticais. Os prolongamentos horizontais das interseções das linhas verticais com a curva $g(T)$ para o eixo vertical, indicam os intervalos das incertezas de $g$. A linha tracejada tangente à curva $g(T)$ indica a derivada $g^{\prime}(T)$. Embora $g(L, T)$ seja não-linear em $T$, para intervalos pequenos em torno de $\bar{T}$ as duas metodologias são equivalentes, ou seja, as linhas contínua $g(T)$ e tracejada $g^{\prime}(T)$ se superpõem quase perfeitamente (Figura 2).

Para resultados que requerem até um ou dois algarismos significativos na incerteza propagada, como é o caso encontrado nos laboratórios de ensino, não haverá diferença entre os métodos. Haverá potencial

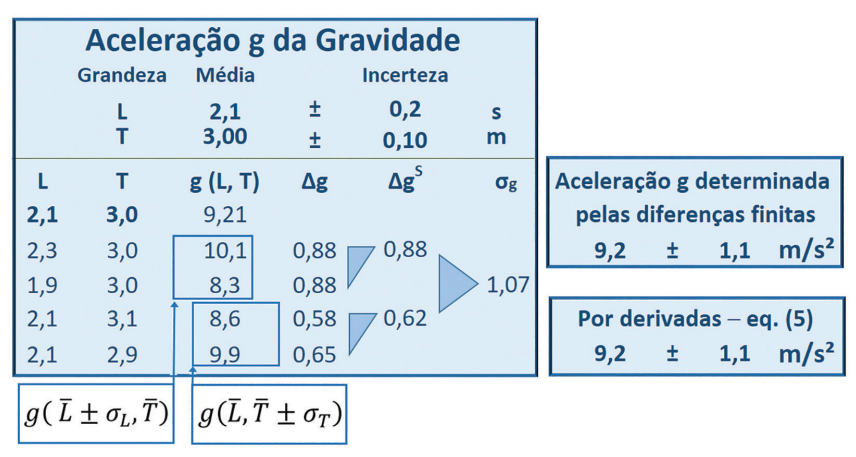

Figura 1: Propagação de incertezas de $g$ obtidas pelo método das diferenças finitas e pelo método convencional das derivadas. Valores medidos: $L=(2,1 \pm 0,2) \mathrm{m}$, e $T=(3,00 \pm 0,10) \mathrm{s}$, onde as incertezas representam $1 \mathrm{DP}$, e $\mathrm{N}=80$ leituras foram usadas para cada medição. Note os passos intermediários conforme a Tabela 1 . Os valores em negrito no quadro acima estão arredondados para apresentar o número correto de algarismos significativos. Observe que as duas metodologias dão o mesmo resultado.

\footnotetext{
3 Devido à distribuição estatística das leituras, inerente ao processo de medida, e ao fato de fazermos um número finito de leituras, numa repetição do experimento tanto a estimativa do valor médio quanto a do desvio padrão mudarão um pouco. A incerteza da incerteza é devida a essa variabilidade na estimativa do desvio padrão de um experimento de medida para outro.
} 


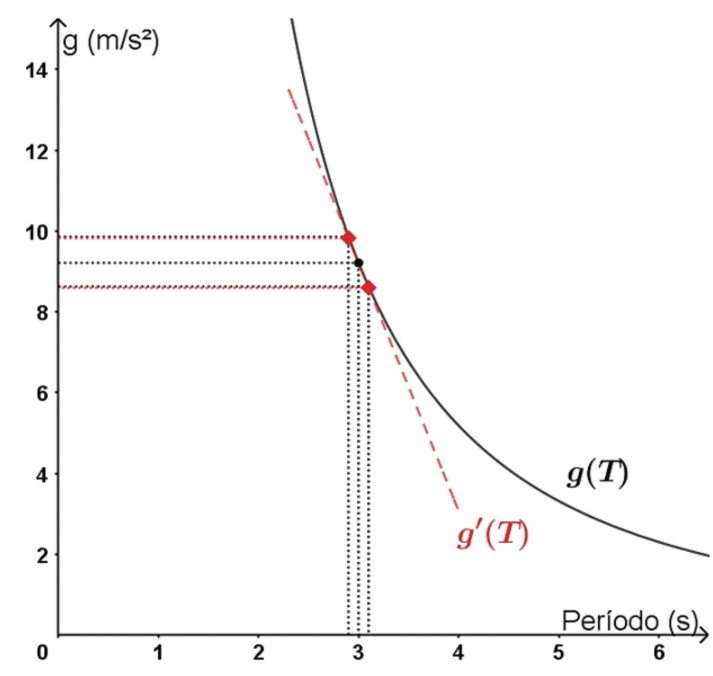

Figura 2: Exemplo de propagação de incertezas de uma variável $T$ na função $g(\bar{L}, T)$, para $T=(3,00 \pm 0,10) \mathrm{s}$, onde a incerteza representa $1 \mathrm{DP}$, e $\bar{L}=2,1 \mathrm{~m}$. Note que para a incerteza em $\bar{T}$ relativamente pequena como mostrada, as projeções $g(T)$ e $g^{\prime}(T)$ são aproximadamente idênticas, levando a mesma incerteza em $g$ quando poucos algarismos significativos são usados.

discrepância quando as incertezas forem maiores que cerca de $10 \%$ do valor médio da variável correspondente. Nesse caso, as curvas $g^{\prime}(T)$ e $g(T)$ diferem entre si ao redor de $\bar{T}$, como pode ser inferido da Figura 2 Porém, o tratamento de grandes incertezas experimentais não pertence ao laboratório introdutório de ensino, e fica fora do escopo deste artigo.

As limitações no uso da equação (9) e da equação (5) são as mesmas: o experimento precisa ter sido planejado de tal forma que as leituras das várias variáveis devem ser feitas para serem independentes e descorrelacionadas, e as incertezas relativas devem ser menores que cerca de $10 \%$.

\section{Considerações finais}

O uso de diferenças finitas na propagação de incertezas visa diminuir a carga cognitiva [17 19 do aluno do laboratório de ensino de física experimental. Nossa abordagem é adequada após o aluno ter entendido e dominado a propagação de incerteza, e permite a análise dos dados de forma relativamente sofisticada, usando planilhas de autoria própria.

\section{Agradecimentos}

RPRF agradece apoio do programa unificado de bolsas de estudo para estudantes de graduação (PUB - USP). Agradecemos a Renata Pazzini e ao Eduardo L. Dias pela leitura crítica do manuscrito.

\section{Material suplementar}

Exemplos da metodologia implementada estão disponíveis em: https://github.com/gccardoso/incerte zas.git

\section{Referências}

[1] E.M. Smith, M.M. Stein, C. Walsh e N. Holmes, Physical Review X 10, 011029 (2020).

[2] R.J. Moffat, Experimental thermal and fluid science 1, 3 (1988).

[3] J.H. Vuolo, Fundamentos da teoria de erros (Editora Blucher, São Paulo, 1996).

[4] O.A. Helene e V.R. Vanin, Tratamento estatístico de dados em física experimental (Editora Blucher, São Paulo, 1991).

[5] F. Marineli e J.L.A. Pacca, Revista Brasileira de Ensino de Física 28, 497 (2006).

[6] T.S. Volkwyn, S. Allie, A. Buffler e F. Lubben, Physical Review Special Topics-Physics Education Research 4, 010108 (2008).

[7] R.L. Kung, American Journal of Physics, 73, 771 (2005).

[8] J. de Pinho Alves Filho, Caderno brasileiro de ensino de Física 17, 174 (2000).

[9] N. Holmes, J. Olsen, J.L. Thomas e C.E. Wieman, Physical Review Physics Education Research 13, 010129 (2017).

[10] G.L. de Sousa e G.C. Cardoso, Physics Education 53 , 055001 (2018).

[11] R.D. Knight, Física-1: Uma Abordagem EstratégicaMecânica Newtoniana, Gravitação, Oscilações e Ondas (Editora Bookman, São Paulo, 2000).

[12] L. Flansburg, American Journal of Physics 40, 1607 (1972).

[13] B. Priemer e J. Hellwig, International Journal of Science and Mathematics Education 16, 45 (2018).

[14] D.C. Bailey, Royal Society open science 4, 160600 (2017).

[15] JCGM, Evaluation of measurement data - Guide to the expression of uncertainty in measurement, Int. Organ. Stand. Geneva 50, 134 (2008).

[16] INMETRO, Avaliação de dados de medição - Guia para a expressão de incerteza de medição - GUM 2008. Disponível em: http://www.inmetro.gov.br/inovacao/ publicacoes/gum_final.pdf

[17] J.J. Van Merriënboer, P.A. Kirschner e L. Kester, Educational psychologist 38, 5 (2003).

[18] A. Buffler, S. Allie e F. Lubben, The Physics Teacher 46, 539 (2008).

[19] J.J. Van Merriënboer e P.A. Kirschner, Ten steps to complex learning: A systematic approach to four-component instructional design (Routledge, Londres, 2017). 